Letter to the Editor

\title{
Pulmonary embolism and COVID-19 pneumonia: the role of non-enhanced chest computed tomography
}

\author{
Payam Mehrian ${ }^{1}$, Pooya Iranpour ${ }^{2}$, Sara Haseli ${ }^{1}$ and Neda Khalili ${ }^{3, *}$
}

${ }^{1}$ Chronic Respiratory Diseases Research Center, National Research Institute of Tuberculosis and Lung Diseases (NRITLD),

Shahid Beheshti University of Medical Sciences, 19839-63113, Tehran, Iran

${ }^{2}$ Medical Imaging Research center, Department of Radiology, Shiraz University of Medical Sciences, 71348-14336, Shiraz, Iran

${ }^{3}$ School of Medicine, Tehran University of Medical Sciences, 14155-6559, Tehran, Iran

*Correspondence: nedakhalili44@gmail.com (Neda Khalili)

DOI: $10.31083 /$ i.rcm.2020.04.246

This is an open access article under the CC BY 4.0 license (https://creativecommons.org/licenses/by/4.0/).

\section{Dear Editor,}

Pulmonary embolism (PE) is a life-threatening clinical condition that is recognized as the third most common cause of cardiovascular death (Turetz et al., 2018). The relatively high mortality related to pulmonary embolism is partly due to delayed or misdiagnosis owing to its non-specific presenting symptoms; therefore, a timely and accurate diagnosis, which is highly dependent on imaging techniques, is crucial in reducing mortality (Moore et al., 2018). Computed tomography pulmonary angiography (CTPA) is currently the gold standard imaging modality for diagnosing PE (Moore et al., 2018). While acute PE could be readily diagnosed as a central filling defect surrounded by intravascular contrast, known as the polo mint and railway track signs on different imaging planes, some of the parenchymal changes that occur in the setting of acute PE are more or less non-specific. For example, pulmonary infarcts typically manifest as a wedge-shaped peripheral opacity (Wittram et al., 2004). Moreover, the atoll sign or reversed halo sign, characterized by a central zone of groundglass opacity surrounded by a rim of dense consolidation, has been described in cases of acute PE as well as in several other lung pathologies (Almeida et al., 2020). Although a confident diagnosis can usually be made using the constellation of the mentioned radiologic findings on contrast-enhanced chest CT scan, making a correct diagnosis may be more challenging when dealing with non-contrast $\mathrm{CT}$ images. In fact, many patients with respiratory signs and symptoms initially undergo a non-enhanced radiologic study. This is particularly the case for many patients with coronavirus disease-2019 (COVID-19) pneumonia in whom a first-line non-contrast chest $\mathrm{CT}$ is performed according to national and international guidelines (Mahdavi et al., 2020; Simpson et al., 2020).

Recently, Jalaber et al. (2020) indicated that there is no clinical benefit in performing routine CTPA at initial screening in unselected COVID-19 patients; thus, CTPA might be best not to be performed systematically in all cases of COVID-19. Although to date most studies have used CTPA for the diagnosis of PE, only about $35 \%$ of patients with COVID-19 have undergone CTPA (Roncon et al., 2020). Nopp and colleagues reported that the COVID-19 pandemic lockdown caused a significant decrease in the number of diagnostic imaging tests (CTPA or V/Q lung scan) for suspected $\mathrm{PE}$ as compared to the corresponding periods in 2018 and 2019 in Austria (Nopp et al., 2020). Deeming the infeasibility of CTPA application in all COVID-19 cases during the height of the current pandemic, identifying patients with the highest index of suspicion for PE seems necessary for determining those in whom CTPA should be considered. On the other hand, it is also important to note that CTPA may result in the overdiagnosis of PE (Hutchinson et al., 2015) and might be associated with several potential harms, including the risk of in-hospital transmission of COVID-19, contamination of CT scanners, contrast-induced nephrotoxicity, unnecessary radiation and allergic reactions.

Peripheral opacities, including ground-glass opacities, consolidations and the atoll sign are among the most important radiologic findings of pneumonia caused by severe acute respiratory syndrome coronavirus-2 (SARS-CoV-2) (Simpson et al., 2020). Also, current evidence affirms the high prevalence of thromboembolic events, specifically PE, in patients with COVID-19 (Bikdeli et al., 2020; Klok et al., 2020); hence, care must be taken when interpreting peripheral opacities, especially the reversed halo sign, on chest CT images of those infected with the novel betacoronavirus. Herein, we present several imaging findings on non-contrast chest $\mathrm{CT}$ that can be suggestive of acute PE, and could thus prompt physicians to perform CTPA for confirmation of PE in COVID19 patients.

Firstly, careful attention to the intraluminal density of a pulmonary vessel may reveal a slight increase in the focal attenuation of the vessel, marked by a hyperattenuating finding, compared with other parts. This is observed in the setting of acute PE on noncontrast CT scan, and can be explained by the higher concentration of hemoglobin in a retracting clot, commonly seen within the first seven days of PE (Morita et al., 2010; Torres et al., 2020).

Secondly, although the atoll sign has been classified as a typical imaging feature of COVID-19 pneumonia, it is usually detected in 
the later phases of disease (Simpson et al., 2020). On the contrary, in a recently published paper, Almeida et al. (2020) concluded that the reversed halo sign is more frequently observed in the initial scans of cases with pulmonary septic emboli and lung infarction. In $\mathrm{PE}$, the underlying pathophysiology of this finding is the ischemic and hemorrhagic changes of the lung parenchyma -as opposed to organizing pneumonia that is the contributing mechanism of lung injury in COVID-19- which possibly explains the different temporal radiologic manifestations. However, further studies are needed to draw a definite conclusion.

In addition, several other ancillary findings on non-contrast chest CT, including dilatation of the pulmonary trunk and also markers suggestive of right heart strain may point out the possibility of PE (Castañer et al., 2006).

Conclusively, as radiologic findings of COVID-19 pneumonia may overlap with those of PE on non-contrast-enhanced chest CT, meticulous attention to the mentioned imaging markers, temporal radiologic changes, and clinical and laboratory parameters is mandatory. In complex clinical scenarios, evaluation by CTPA is the key to diagnosis, preventing further mortality and morbidity. Nevertheless, the decision to perform CTPA should be made if the benefits clearly outweigh its potential harms, since CT examination should be performed with the least possible harm to the patient. Until now, an established cut-off value for D-dimer has not been detected and also the relevance of conventional PE probalility scores (Wells criteria, Geneva score, and Pulmonary Embolism Rule-out Criteria) has not been studied in COVID-19 (Espallargas et al., 2020). As suggested by the pulmonary embolism response team (PERT) guideline (Rosovsky et al., 2020), a modified algorithm for the diagnosis and management of PE should be developed in this time period.

\section{Abbreviations}

COVID-19, coronavirus disease-2019; CTPA, computed tomography pulmonary angiography; PE, pulmonary embolism; PERT, pulmonary embolism response team; SARS-CoV-2, severe acute respiratory syndrome coronavirus- 2 .

\section{Author's contributions}

All authors have participated in study design and writing of the manuscript, and have read and approved the final manuscript.

\section{Acknowledgements}

We would like to express our gratitude to all those who helped us during the writing of this manuscript.

\section{Funding}

This research did not receive any specific grant from funding agencies in the public, commercial, or not-for-profit sectors.

\section{Conflict of interest}

The authors declare that they have no conflict of interest.

\section{References}

Almeida, R. R., Marchiori, E. and Flores, E. J. (2020) Frequency and reliability of the reversed halo sign in patients with septic pulmonary embolism due to IV substance use disorder. American Journal of Roentgenology 214, 59-67.

Bikdeli, B., Madhavan, M. V., Jimenez, D., Chuich, T., Dreyfus, I., Driggin, E., Nigoghossian, C., Ageno, W., Madjid, M., Guo, Y., Tang, L. V., Hu, Y., Giri, J., Cushman, M., Quéré, I., Dimakakos, E. P., Gibson, C. M., Lippi, G., Favaloro, E. J., Fareed, J., Caprini, J. A., Tafur, A. J., Burton, J. R., Francese, D. P., Wang, E. Y., Falanga, A., McLintock, C., Hunt, B. J., Spyropoulos, A. C., Barnes, G. D., Eikelboom, J. W., Weinberg, I., Schulman, S., Carrier, M., Piazza, G., Beckman, J. A., Steg, P. G., Stone, G. W., Rosenkranz, S., Goldhaber, S. Z., Parikh, S. A., Monreal, M., Krumholz, H. M., Konstantinides, S. V., Weitz, J. I. and Lip, G. Y. H. (2020) COVID-19 and thrombotic or thromboembolic disease: implications for prevention, antithrombotic therapy, and follow-up: JACC state-of-the-art review. Journal of the American College of Cardiology 75, 2950-2973.

Castañer, E., Gallardo, X., Rimola, J., Pallardó, Y., Mata, J. M., Perendreu, J., Martin, C. and Gil, D. (2006) Congenital and acquired pulmonary artery anomalies in the adult: radiologic overview. RadioGraphics $\mathbf{2 6}$, 349-371.

Espallargas, I., Rodríguez Sevilla, J. J., Rodríguez Chiaradía, D. A., Salar, A., Casamayor, G., Villar-Garcia, J., Rodó-Pin, A., Marsico, S., Carbullanca, S., Ramal, D., del Carpio, L. A., Gayete, Á., Maiques, J. M. and Zuccarino, F. (2020) CT imaging of pulmonary embolism in patients with COVID-19 pneumonia: a retrospective analysis. European Radiology (in press).

Hutchinson, B. D., Navin, P., Marom, E. M., Truong, M. T. and Bruzzi, J. F. (2015) Overdiagnosis of pulmonary embolism by pulmonary CT angiography. American Journal of Roentgenology 205, 271-277.

Jalaber, C., Revel, M. P., Chassagnon, G., Bajeux, E., Lapotre, T., Croisille, P. and Lederlin, M. (2020) Role of upfront CT pulmonary angiography at admission in COVID-19 patients. Thrombosis Research 196, 138-140.

Klok, F. A., Kruip, M. J. H. A., van der Meer, N. J. M., Arbous, M. S., Gommers, D. A. M. P. J., Kant, K. M., Kaptein, F. H. J., van Paassen, J., Stals, M. A. M., Huisman, M. V. and Endeman, H. (2020) Incidence of thrombotic complications in critically ill ICU patients with COVID19. Thrombosis Research 191, 145-147.

Mahdavi, A., Khalili, N., Davarpanah, A. H., Faghihi, T., Mahdavi, A. Haseli, S., Sabri, A., Kahkouee, S., Kazemi, M. A., Mehrian, P., Falahati, F., Bakhshayeshkaram, M. and Sanei Taheri, M. (2020) Radiologic management of COVID-19: preliminary experience of the iranian society of radiology COVID-19 consultant group (ISRCC). Iranian Journal of Radiology 17, e102324.

Moore, A. J. E., Wachsmann, J., Chamarthy, M. R., Panjikaran, L., Tanabe, Y. and Rajiah, P. (2018) Imaging of acute pulmonary embolism: an update. Cardiovascular Diagnosis and Therapy 8, 225-243.

Morita, S., Ueno, E., Masukawa, A., Suzuki, K., Machida, H. and Fujimura, M. (2010) Hyperattenuating signs at unenhanced ct indicating acute vascular disease. RadioGraphics 30, 111-125.

Nopp, S., Janata-Schwatczek, K., Prosch, H., Shulym, I., Königsbrügge, O., Pabinger, I. and Ay, C. (2020) Pulmonary embolism during the COVID-19 pandemic: decline in diagnostic procedures and incidence at a university hospital. Research and Practice in Thrombosis and Haemostasis 4, 835-841.

Roncon, L., Zuin, M., Barco, S., Valerio, L., Zuliani, G., Zonzin, P. and Konstantinides, S. V. (2020) Incidence of acute pulmonary embolism in COVID-19 patients: systematic review and meta-analysis. European Journal of Internal Medicine (in press). 
Rosovsky, R. P., Grodzin, C., Channick, R., Davis, G. A., Giri, J. S., Horowitz, J., Kabrhel, C., Lookstein, R., Merli, G., Morris, T. A., Rivera-Lebron, B., Tapson, V., Todoran, T. M., Weinberg, A. S. and Rosenfield, K. (2020) Diagnosis and treatment of pulmonary embolism during the COVID-19 pandemic: a position paper from the national PERT consortium. Chest (in press).

Simpson, S., Kay, F. U., Abbara, S., Bhalla, S., Chung, J. H., Chung, M., Henry, T. S., Kanne, J. P., Kligerman, S., Ko, J. P. and Litt, H. (2020) Radiological society of north America expert consensus statement on reporting chest CT findings related to COVID-19. Endorsed by the society of thoracic radiology, the American college of radiology, and RSNA. Radiology 2, e200152.
Torres, P., Mançano, A. D., Zanetti, G., Hochhegger, B., Aurione, A. C. V., Rabahi, M. F. and Marchiori, E. (2020) Multimodal indirect imaging signs of pulmonary embolism. British Journal of Radiology 93, 20190635.

Turetz, M., Sideris, A. T., Friedman, O. A., Triphathi, N. and Horowitz, J. M. (2018) Epidemiology, pathophysiology, and natural history of pulmonary embolism. Seminars in Interventional Radiology 35, 92-98.

Wittram, C., Maher, M. M., Yoo, A. J., Kalra, M. K., Shepard, J.-A. O. and McLoud, T. C. (2004) CT angiography of pulmonary embolism: diagnostic criteria and causes of misdiagnosis. RadioGraphics 24, 12191238. 